\title{
Energy Efficient Resource Allocation in Cloud Computing
}

\author{
Ch VijayaKumari, M Aharonu, T. Sunil
}

\begin{abstract}
Cloud service provider in cloud environment will provide or provision resource based on demand from the user. The cloud service provider (CSP) will provide resources as and when required or demanded by the user for execution of the job on the cloud environment. The CSP will perform this in a static and dynamic manner. The CSP should also consider various other factors in order to provide the resources to the user, the prime among that will be the Service Level Agreement (SLA), which is normally signed by the user and cloud service provider during the inception phase of service. There are many algorithm which are used in order to allocate resources to the user in cloud environment. The algorithm which is proposed will be used to reduce the amount of energy utilized in performing various job execution in cloud environment. Here the energy utilized for execution of various jobs are taken into account by increasing the number of virtual machines that are used on a single physical host system. There is no thumb rule to calculate the number of virtual machines to be executed on a single host. The same can be derived by calculating the amount of space, speed required along with the time to execute the job on a virtual machine. Based up on this we can derive the number of Virtual machine on a single host system. There can be 10 virtual machines on a single system or even 20 number of virtual machines on single physical system. But if the same is calculated by the equation then the result will be exactly matching with the threshold capacity of the physical system [1]. If more number of physical systems are used to execute fewer virtual machines on each then the amount of energy consumed will be very high. So in order to reduce the energy consumption , the algorithm can be used will not only will help to calculate the number of virtual machines on single physical system, but also will help to reduce the energy as less number of physical systems will be in need[2].

Keywords:
\end{abstract}

Virtual, machine, energy, physical, host, system, SLA.

\section{INTRODUCTION :}

Usage of various services such as software service, platform services and infrastructure service or hardware service in order to make an application run on internet from an external

source can be refereed as Cloud computing as in Fig: 2 . The user here can makes use of these resources and can pay to the cloud service provider as per use. It is like a utility service such as an electricity bill and telephone bill as shown in Fig: 1 . The user here can enter into an agreement with the cloud service provider to make use of resource as and when required[3].

Revised Manuscript Received on 14 September, 2019.

Mrs. Ch VijayaKumari, Department of CSE, Malla Reddy College of Engineering, Hyderabad India

Mr. M Aharonu, Department of CSE, Malla Reddy College of Engineering, Hyderabad India

Dr. T. Sunil Department of CSE, Malla Reddy College of Engineering, Hyderabad India.
This agreement is referred as Service Level Agreement (SLA).

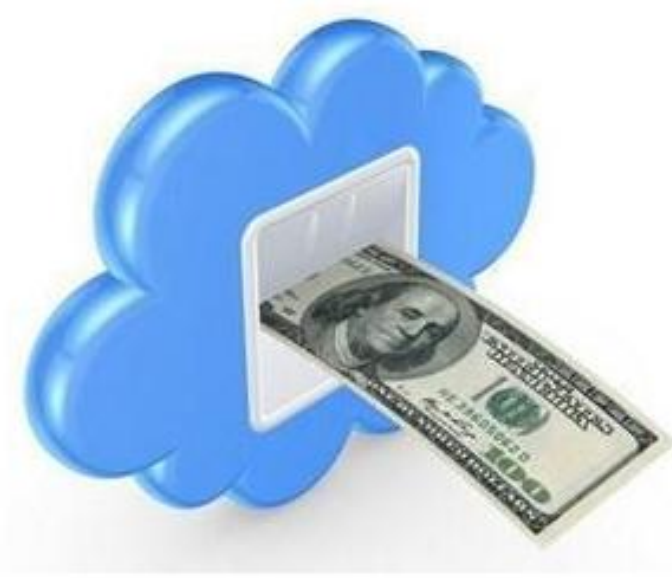

Fig:1

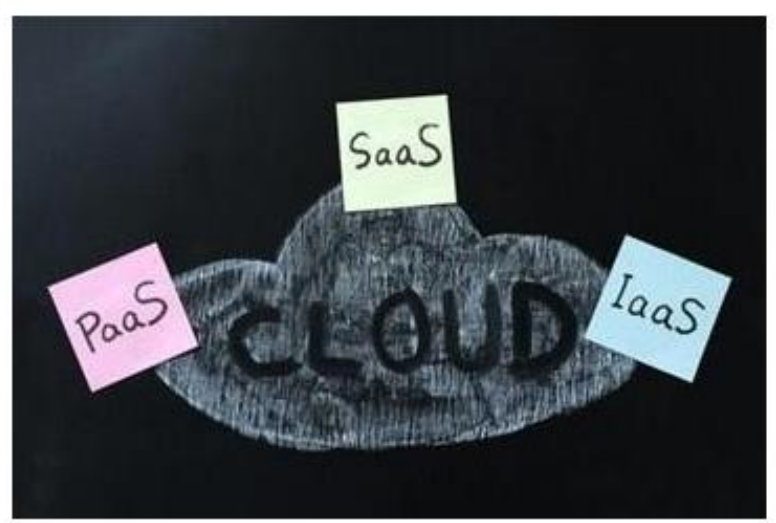

Fig:2

Virtualization is a process where on a physical system a virtual environment can be created so as to run a desired program by the user as in Fig:4[1]. We can use this concept of virtualization for hardware virtualization, software and network virtualization. Coming to hardware virtualization there can be many subtypes in using this concept such as Full virtualization, Para virtualization and Emulation virtualization. Likewise subtypes also exists in software virtualization such as virtualization in Service called as service virtualization, Application virtualization and Operating system virtualization as in Fig: 3. In memory virtualization there can be two subtypes like Application level control and operating system level control. Further storage virtualization can also be at block level virtualization and file level virtualization[4].

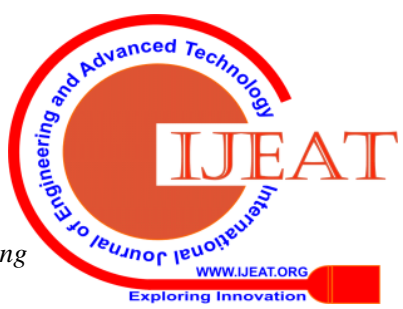




\section{Energy Efficient Resource Allocation in Cloud Computing}

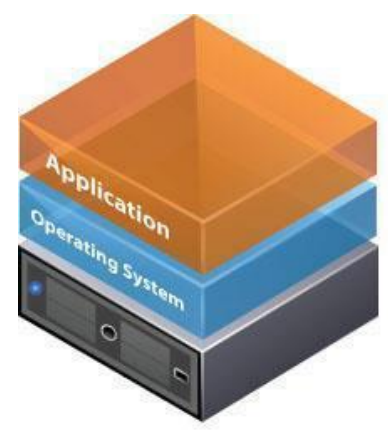

Traditional Architecture

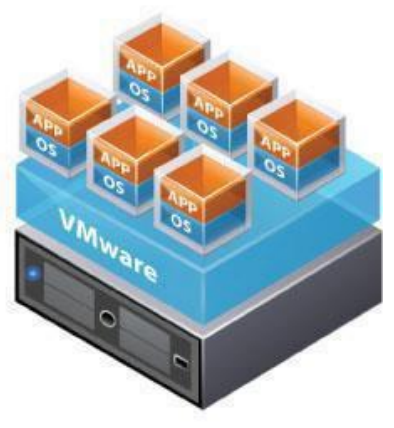

Virtual Architecture
Fig:3

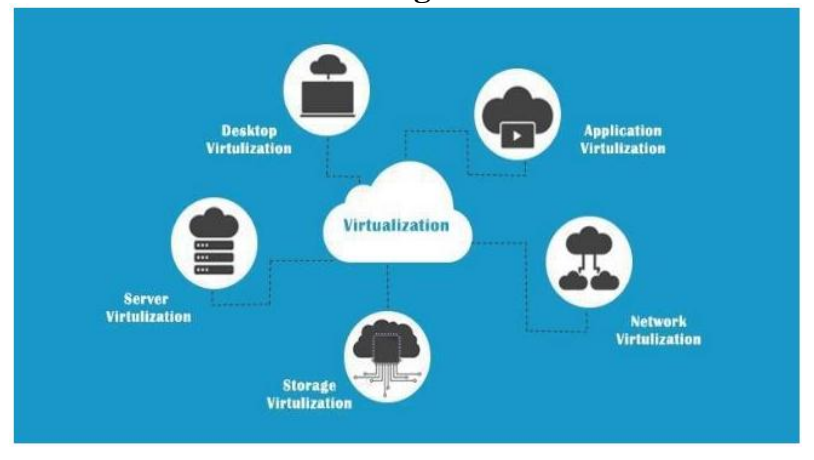

Fig:4

Access to the virtual machine and the host machine/ server is facilitated by a software known as Hypervisor. Hypervisor acts as a link between the hardware and the virtual environment and distributes the hardware resources such as CPU usage, memory allotment between the different virtual environments[5].

\section{RELATED WORK:}

We normally use cloud services for storing the data, photos, and videos, documents using Amazon services or Microsoft Services or even google services for that matter. When cloud services are used infrequently the people performing research found that the power consumed is far less when it is compared with the power

consumption in conventional computing and storage. Researchers found that the at low levels of usage the power consumption for storage of data and other things will dominate the total consumption of power as the power for transport is minimal or minimum. This is not true when the usage levels are at medium and high levels, more amount of energy is required to transmit data and because of which total consumption of power increases drastically over the overall energy consumed[6].

More precisely the power consumption for transmission of data can be very low for low usage of levels for public and private storage which can be $10 \%$ to $25 \%$ and the same will increase drastically for high usage levels and will be around $60 \%$ and $90 \%$ [7].

From this it is very clear that the cloud services when utilized for storage will use less amount of energy compared to cloud usage for processing of data and used for cloud computing. As long as the screen refresh rates are very low like say 0.1 frames/sec the amount of power consumption in transport is small in Cloud software services such as Google Documents, where as one 1 frame/sec means that $100 \%$ changes happens on the screen every second

and so. Smaller the percentage change in the screen will reflect in low or smaller refresh rate. Biggest or important factor for the cloud software services where energy is to be determined working on a particular server. So in case like this the more beneficial one will be the public cloud users when compared with private cloud users because of its larger number of users working[10]. For cloud handling administrations (in which a server, for example, Amazon Elastic Compute Cloud forms extensive computational assignments just, and littler undertakings are prepared on the client's PC), the scientists again discovered that the cloud option can utilize bring down utilization just under specific conditions. The outcomes demonstrated that, for open cloud preparing administrations, information transport devoured a lot of vitality contrasted with private cloud handling administrations, especially at high utilization levels. The reason is that the vast number of switch bounces required on the general population Internet incredibly builds the vitality utilization in transport, and private cloud preparing requires fundamentally less switches[8]. All things considered, the analysts found that, for both open and private mists, a cloud preparing administration is more vitality proficient than more seasoned age PCs. The overall results specify different concept for different type of users. As the research performed by the group states that the computer user at home can achieve significant amount of energy savings by making use of low end computers or laptops for performing the task which are of routine nature where as to perform the task occasionally which are of high computational nature the use of cloud service can be made use of instead of making use of high end PC or a mid-range PC[9].

For companies, it is less certain whether the vitality utilization spared in transport with a private cloud contrasted with an open cloud counterbalances the private cloud's higher vitality utilization[6]. Private mists that serve a generally modest number of clients may not profit by a similar vitality sparing methods because of their littler scale[10].

As distributed computing turns out to be progressively across the board it is trusted that or expectations are to such an extent that the distributed computing will turn out to be increasingly more vitality proficient in information transport, particularly with the server farms where gadgets like ,switches ,centre points are utilized. As we know that the device which are used to transfer data from one place to another will have less or limited capacity, it is then the only solution available for the user to have data mobility is by making use of cloud services. Which most of the industry people believe and started working using cloud computing concepts. The client gadget will contain minimal in excess of a program when it is begun up. Any application or information that it requires will be brought down from the cloud[11]. At the point when that application is done, its information will be returned to the cloud and the application will be expelled from the client gadget until the point that it is again required. Along these lines, the client gadget is kept basic, vitality effective and shoddy." Various parts of asset the executives influence server control utilization? Understanding the impact of various parameters on power utilization is essential so as to oversee servers in a vitality productive way.

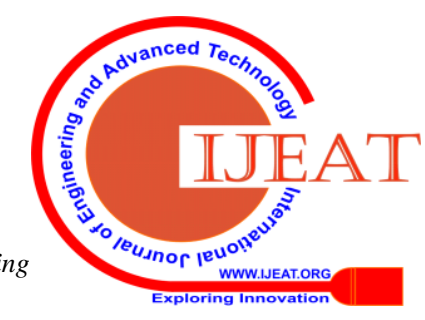


These parameters may incorporate differing the quantity of virtual machines (VMs), fluctuating the figuring assets allotted to a VM, (for example, CPU centres and memory), and turning centres on/off, shifting the CPU recurrence and mapping virtual CPUs to physical CPU centres.

proposed algorithm:

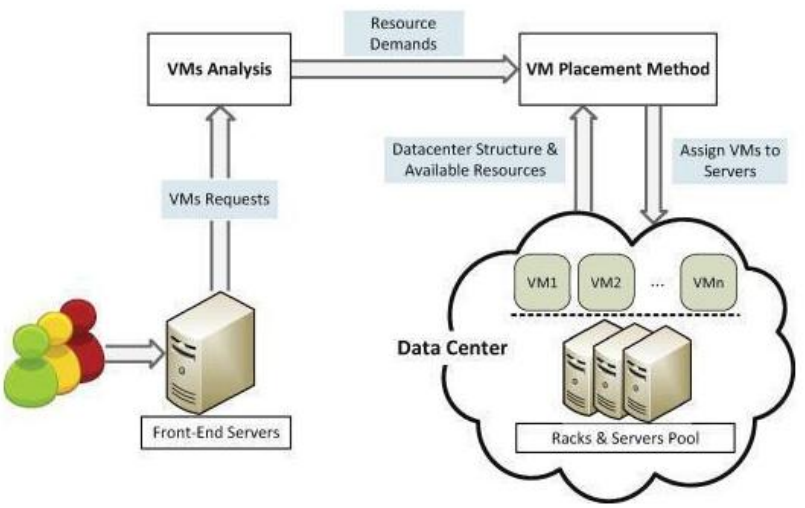

Here we have consider the fact that the number of physical systems used will be directly proportional to the number of physical servers reaching the threshold capacity. i.e. to say if the number of physical systems used are ten, it indicates that the nine physical systems have reached the threshold capacity so the need for usage of tenth system has arised. If we consider the V1, V2, and V3 ... As the virtual

machines and $\operatorname{TrC}$ as the threshold capacity of the physical system then

$\sum_{1} \quad 1,2,3 \ldots<=$ and if the
$\sum \quad 1,2,3 \ldots>=$ then it is an indication that the next physical system has to be used for execution of various virtual machines.

If $P 1, P 2, P 3 \ldots$ are the physical systems and the power utilised by $P 1, P 2 \ldots$ is $P o 1, P o 2$ and so on then the number of systems reduced will be equal to the amount of power consumption reduction in the data centre.

Algorithm:

Begin

for each job in incoming queue

allocate virtual machine if the sum of

$v m s>=\operatorname{Tr} C$

allocate VM on new physical system

else

allocate VM on existing physical system

for-each

Experimental Results:

$\operatorname{Tr} C S 1=M(v 1+v 2+v 3+v 4)$

$\operatorname{Tr} C S 2=M(v 1+v 2+v 3+v 4)$

$\operatorname{Tr} C S 3=M(v 1+v 2+v 3+v 4)$

$\operatorname{Tr} C S 4=M(v 1+v 2+v 3+v 4)$
Threshold capacity of the System one is calculated as maximum capacity of virtual machine1, virtual machine2 virtual machine3, virtual machine4.

So if S4 is started without the threshold of S3 is reached then it will consume more energy than what is required.Which means the enery consumed per se is 1 unit per system then we have to consume 4 units intead of three units to complete the task given.

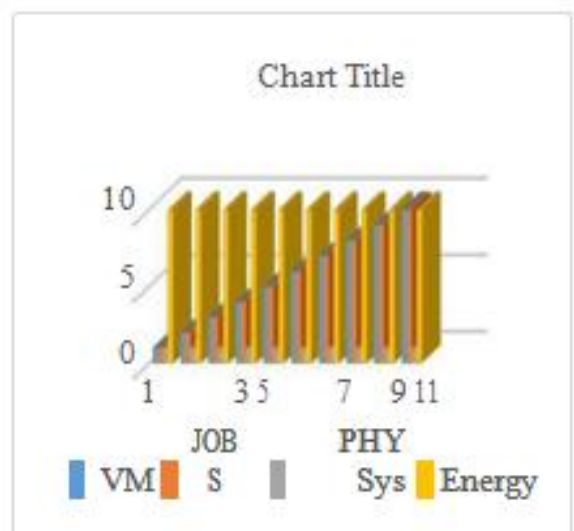

Graph:1
PSystem

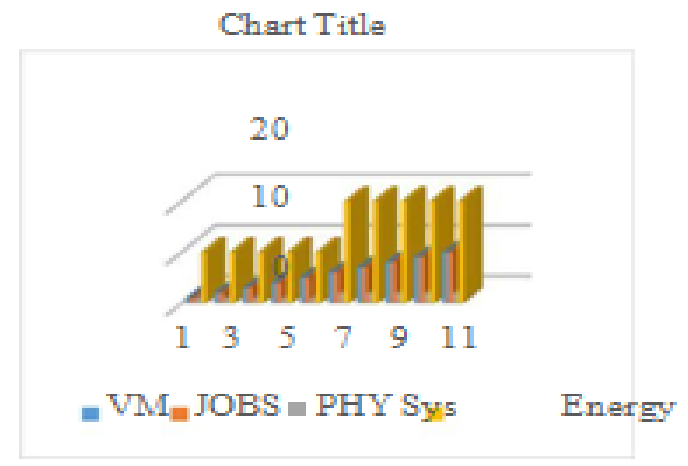

Graph:2 Energy Consumption with two PSystem

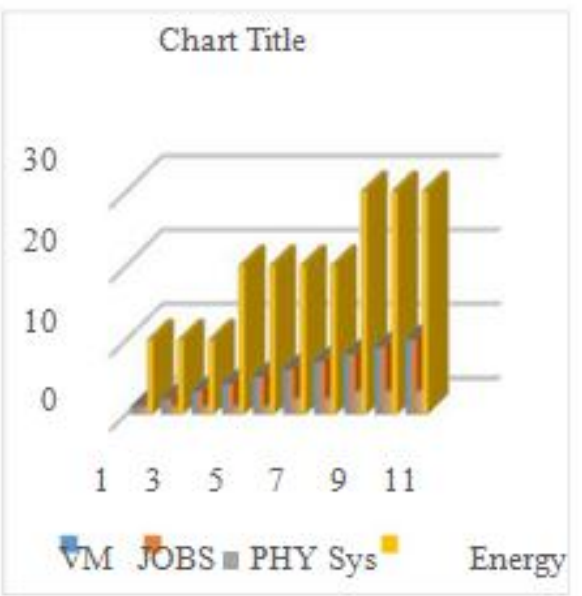

Graph:3 Energy Consumption with three PSystem

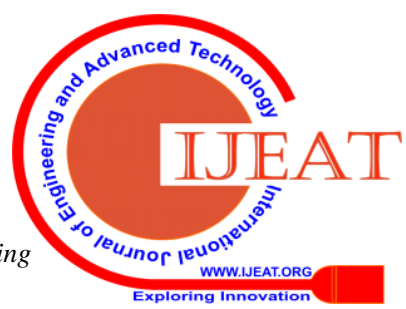


So in the above graph it is observed that if the virtual machines are used till the threshold capacity of the physical server is reached then the number of physical systems usage will be reduced there by reducing the consumption of energy. For the same number of virtual machines if one physical system is used then the graph:1 shows the energy consumption, where as for same number of virtual machines two or three physical systems are used then graph:2 and graph:3 shows the consumption of energy.

\section{CONCLUSION:}

From the above algorithm and experiment work which is carried out the conslusion is that the number of physical systems to be used should be directly proportional to the number of virtual machines which can be launched on a physical system till the threshold capacity of the physical system is reached. Doing so we can straight away reduced lot of enegry consumption in the data center. Which will make the cloud computing as green computing and will also help the user to save lot on infrastructure utilisation.

\section{REFERENCES:}

1. D.Saranya;Balancing Load Algorithms in CC.

2. A Review International Journal of Advanced Research in Computer Science and Software Engineering, vol. 5, Issue 7, 2015 July.

3. S. Sethi . Efficient Load Balancing in Cloud Computing usingFuzzy Logic,IOSR Journal of Engineering (IOSRJEN) ISSN: 2250 -3021 vol. 2, pp. 65-71, 2012.

4. 3 T. Desai. A Survey of Various Load Balancing Techniques and Challenges in Cloud Computing. International Journal of Scientific \&amp; Technology Research, vol. 2, Nov 2013.

5. S. Rajoriya. Load Balancing Techniques in Cloud Computing: An Overview, International Journal of Science and Research (IJSR), vol. 3, July 2014

6. Sharma S. et.al, "Performance Analysis of Load Balancing Algorithms," World Academy of Science, Engineering and Technology, 38, 2008.

7. Gross D. et.al, "No cooperative load balancing in distributed systems", Elsevier, Journal of Parallel and Distributed Computing, No. 65, pp. 1022-1034, 2005.

8. Nikravan M. "A Genetic Algorithm for Process Scheduling in Distributed Operating Systems Considering Load Balancing", Proceedings 21st European Conference on Modelling and Simulation (ECMS), 2007.

9. M. Amar "Service level agreement Driven Load Balancing for Web Applications in Cloud Computing Environment",

10. Tian W, Xu M, Chen A. Open-source simulators for cloud computing: Comparative study and challenging issues. 2015:239254.

11. .Sunil Tekale Evaluation based load balancing International Journal of Engineering \& Technology.

12. W Tian Xu M, Y Zhong . A kit for simulation/ modeling of realtime VM allocation in CC. IEEE Trans Autom Sci Eng. 2016 